\title{
Stress Relaxation of Poly-Si Film Formed by Excimer Laser Annealing
}

\author{
Naoto Matsuo \\ Department of Materials Science and Engineering, University of Hyogo, Himeji 671-2280, Japan
}

We investigated the low-temperature processed polycrystalline-silicon (poly-Si) film formed by carrying out low-energy-density and multishot excimer laser annealing (ELA) of amorphous $\mathrm{Si}(\mathrm{a}-\mathrm{Si})$ film on a glass or quartz substrate. The influence of secondary grain growth which grain size becomes larger than the film thickness on the tensile stress relaxation of the poly-Si film was clarified. Relationship between hydrogens which are supplied to the melt-Si from the bottom catalytic-chemical vapor deposition (Cat-CVD) SiN film and the stress relaxation was also examined. The tensile stress in the poly-Si film relaxes upon the appearance of the secondary grains, and the stress decreases with increasing diameter of the secondary grains. However, the hydrogen supply to melt-Si suppresses the secondary grain growth and the stress relaxation becomes marked as the hydrogen concentration decreases. Furthermore, the relationship between the dangling bond density corresponding to the crystal defect density and grain size was investigated, and the defect site was clarified. Lastly, the recrystallization model by ELA at low energy densities was discussed. The model was constructed based on the experimental data that the secondary grain growth occurs suddenly at the critical energy density and the critical shot number, and it was shown that the present model is consistent with the results related to the hydrogen concentration and the secondary grain growth.

(Received April 19, 2005; Accepted July 12, 2005; Published September 15, 2005)

Keywords: tensile stress, polycrystalline silicon, excimer laser annealing, secondary grain growth, hydrogen, dangling bond, catalytic chemical vapor deposition SiN

\section{Introduction}

Recently, silicon film recrystallized by excimer laser annealing (ELA) of amorphous-silicon (a-Si) on a glass substrate has become necessary for use as the materials of the active area of thin-film transistors (TFTs) of liquid-crystal displays (LCDs) or organic light emitting diode (OLED) displays. The requirements for the recrystallized Si film are high quality, which means a film with a low defect density, large grains and position control of nucleation. To satisfy these requirements, sequential lateral solidification (SLS), ${ }^{1)}$ phase-modulated ELA (PMELA), ${ }^{2)}$ multistep $\mathrm{XeCl} \mathrm{ELA},{ }^{3)}$ solid-phase crystallization (SPC) followed by ELA, ${ }^{4)}$ singleshot $\mathrm{XeCl} \mathrm{ELA}^{5}{ }^{5}$ diode-pumped solid-state $\mathrm{CW}$ laser crystallization, ${ }^{6-9)}$ crystallization by a linearly polarized YAG pulse laser ${ }^{10)}$ and the $\mu$-Czochralski method ${ }^{11)}$ have been studied. Field-enhanced metal-induced crystallization (FE-MIC), ${ }^{12)}$ imprinting using $\mathrm{Ni}^{13)}$ and SPC using $\mathrm{Ge}$ and $\mathrm{Si}^{14}$ ) have also been investigated in the low-temperature range. In our group, the mechanism of secondary grain growth by ELA has been examined to provide a high-quality large-grained film. ${ }^{15-26)}$ Here, the secondary grain is defined as having a grain size larger than the film thickness. It was found by electron spin resonance (ESR) measurement that most of the crystal defects were located at the grain boundary of poly-Si film and that hydrogen in a-Si film influenced the crystallinity of the poly-Si. ${ }^{15,16)} \mathrm{We}$ also confirmed that the secondary grain growth of poly-Si film suddenly occurred at the critical energy density and critical shot number. ${ }^{16,17)}$ On the basis of these results, a new crystal growth model was presented. ${ }^{18-21)}$ The purpose of this paper is to review the characteristics of the poly-Si film recrystallized by the ELA method. ${ }^{22-26)}$ First, the influence of the secondary grain growth on the stress relaxation of poly-Si film is explained. Secondly, the effect of the hydrogen concentration on the relation between the tensile stress and grain size of poly-Si film is discussed, referring to the results of experiments with controlled hydrogen concentrations. Thirdly, the defect density in poly-Si film is explained considering the grain size and the hydrogen concentration. Lastly, those phenomena are verified considering our proposed mechanism of secondary grain growth.

\section{Experimental Methods}

The first experiment was conducted to examine the influence of secondary grain growth on the stress relaxation of poly-Si film; SiN film was deposited by plasma chemical vapor deposition (PCVD). The second experiment was conducted to examine the effect of the hydrogen concentration on the relation between the tensile stress and grain size of poly-Si film and on the defect density in poly-Si film. SiN film was deposited by using the catalytic-chemical vapor deposition (Cat-CVD).

\subsection{PCVD SiN film}

An a-Si film $(100 \mathrm{~nm})$ was deposited on $\mathrm{SiO}_{2}(50 \mathrm{~nm}) / \mathrm{SiN}$ $(50 \mathrm{~nm}) /$ glass and $\mathrm{SiO}_{2}(100 \mathrm{~nm}) /$ glass substrates by plasmaenhanced (PE) CVD. An a-Si film was also deposited on a quartz substrate by the low-pressure chemical vapor deposition (LPCVD) method to decrease the hydrogen concentration in a-Si film. The deposition conditions of the PCVD $\mathrm{SiN}$ film are a substrate temperature of $400^{\circ} \mathrm{C}$, an $\mathrm{RF}$ power of $370 \mathrm{~W}$, and an RF frequency of $13.56 \mathrm{MHz}$. Dehydrogenation was carried out by annealing at $450^{\circ} \mathrm{C}$ for $60 \mathrm{~min}$.

\subsection{Cat-CVD SiN film}

To conduct further precise experiments, an a-Si film $(50 \mathrm{~nm})$ was deposited on the $\mathrm{SiN}(50 \mathrm{~nm})$ /quartz substrate by LPCVD. SiN film was successfully formed by the CatCVD method, ${ }^{27,28)}$ and the hydrogen concentrations were fixed at 2.3, 4.2 and 8.2 at $\%$.

\subsection{ELA and analysis of the film}

The KrF multishot excimer laser was irradiated onto the a-Si film at $1 \mathrm{~Hz}$ and at room temperature in vacuum 
(approximately $10^{-4} \mathrm{~Pa}$ ). The pulse duration of the laser was $23 \mathrm{~ns}$. The energy density and shot number ranged from 200 to $500 \mathrm{~mJ} / \mathrm{cm}^{2}$ and 8 to 300 shots, respectively. The internal stress, defect density and surface morphology of the poly-Si film were examined by Raman spectroscopy and scanning electron microscopy (SEM). Raman spectroscopy was carried out with a backscattering geometry and a $514.5 \mathrm{~nm}$ Ar ion laser with a spot size of $0.8 \mu \mathrm{m}$. The full-width at halfmaximum (FWHM) of the transverse optical (TO) Raman peak of the $\operatorname{Si}(100)$ wafer was $4.2 \mathrm{~cm}^{-1}$. The hydrogen in the SiN film was detected by thermal-desorption spectroscopy (TDS). The ion currents were measured using a quadrupole mass spectrometer. The increase rate of temperature, the maximum temperature and the vacuum pressure were $60^{\circ} \mathrm{C} /$ $\min , 600^{\circ} \mathrm{C}$ and $7 \times 10^{-7} \mathrm{~Pa}$, respectively.

\section{Results and Discussion}

\subsection{Influence of secondary grain growth on stress relaxation of poly-Si film}

Figures 1(a)-(c) show the SEM images of poly-Si films on three different substrates where ELA was performed under the conditions of $350 \mathrm{~mJ} / \mathrm{cm}^{2}$ and 100 shots on a quartz substrate, $300 \mathrm{~mJ} / \mathrm{cm}^{2}$ and 100 shots on a $\mathrm{SiO}_{2} /$ glass substrate, and $400 \mathrm{~mJ} / \mathrm{cm}^{2}$ and 100 shots on a $\mathrm{SiO}_{2} / \mathrm{SiN} /$
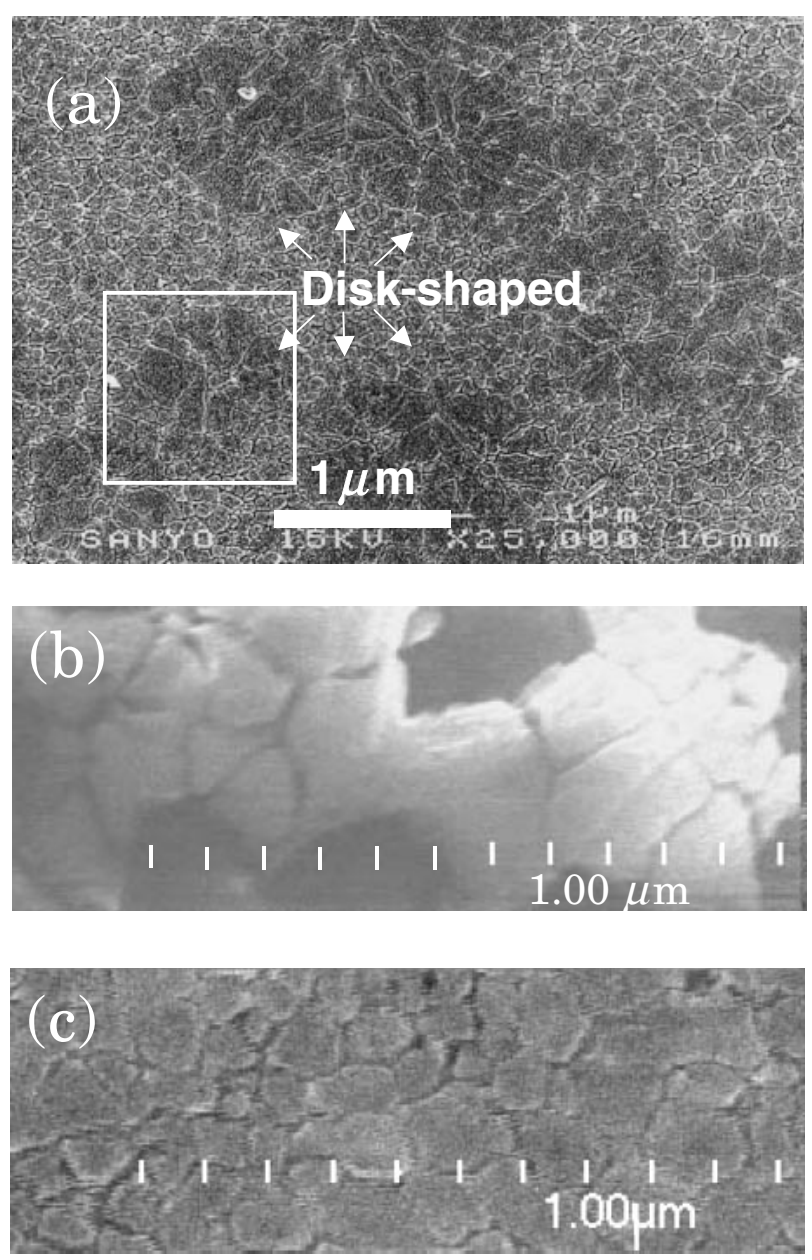

Fig. 1 SEM images of poly-Si films on a quartz substrate (a), on a $\mathrm{SiO}_{2} /$ glass substrate (b) and on a $\mathrm{SiO}_{2} / \mathrm{SiN} /$ glass substrate (c). glass substrate, respectively. Although the secondary grains are not observed, disk-shaped grains ${ }^{19,23,29)}$ and textured grains are observed on the quartz substrate. The secondary grain growth is observed on the $\mathrm{SiO}_{2}$ /glass substrate. The grain size of the poly-Si film on the $\mathrm{SiO}_{2}$ /glass substrate is larger than that of the poly-Si film on the $\mathrm{SiO}_{2} / \mathrm{SiN} /$ glass substrate. For the poly-Si film on the $\mathrm{SiO}_{2} / \mathrm{SiN} /$ glass substrate, only a few secondary grains are observed. Almost all the grains are small. The difference of the morphologies of the poly-Si film between on the quartz substrate and on the $\mathrm{SiO}_{2} /(\mathrm{SiN} /)$ glass substrate is thought to be related with the thermal conductivity of the substrate. Figure 2 shows the relationship between the grain size and the shot number for poly-Si film on each substrate. The average grain size of the textured grains (@) on the quartz substrate is slightly smaller than that $(\square)$ on the $\mathrm{SiO}_{2} / \mathrm{SiN} /$ glass substrate. The average grain size (-) of poly-Si film on the $\mathrm{SiO}_{2} /$ glass substrate is the largest of the poly-Si films on the three types of substrates. Figure 3 shows the FWHM of the TO phonon peak as a function of the Raman peak shift, $\Delta \omega$, for the three

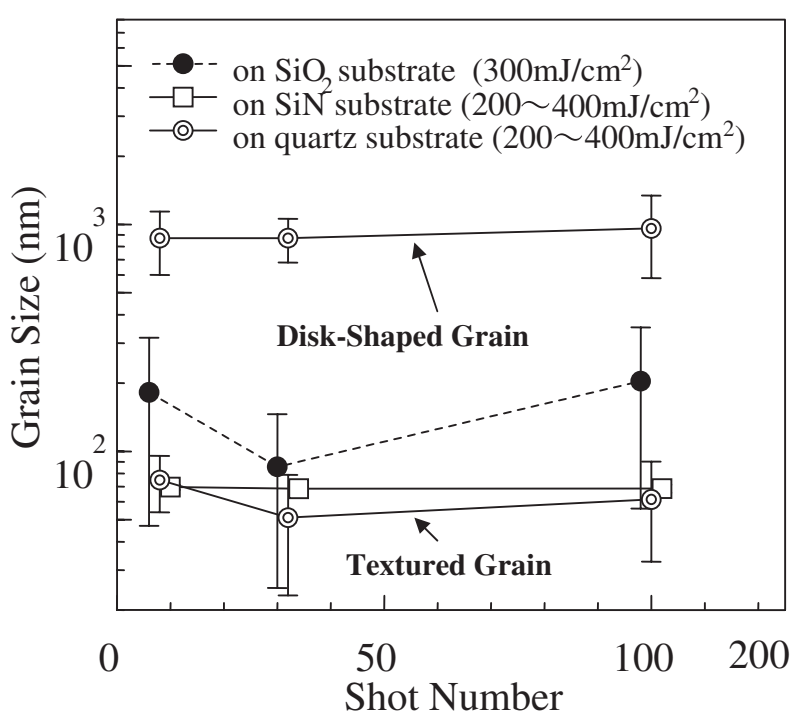

Fig. 2 Relationship between grain size and shot number.

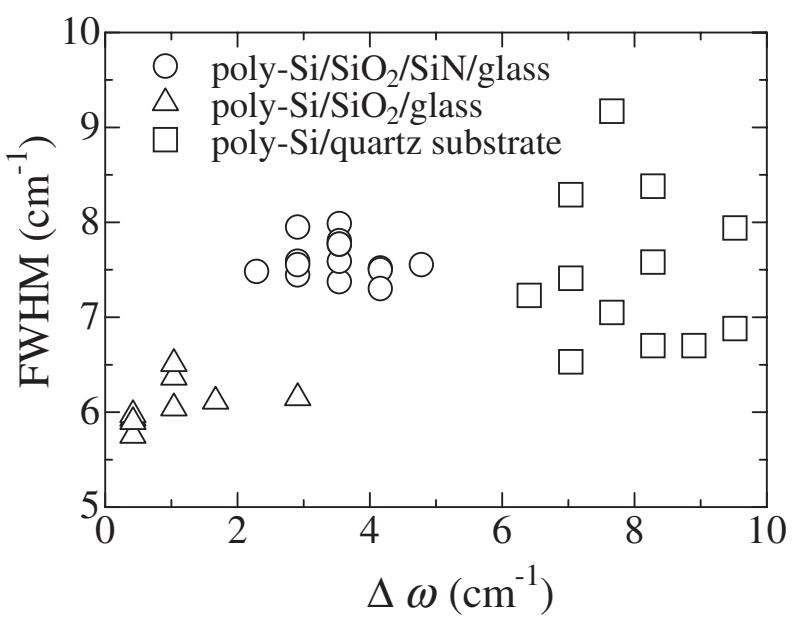

Fig. 3 Relationship between FWHM and $\Delta \omega$ for poly-Si films on each substrate. 


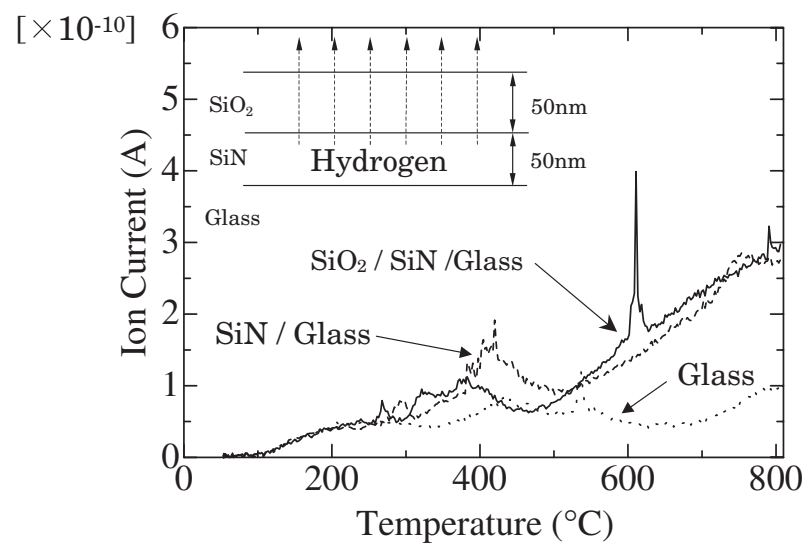

Fig. 4 Relationship between ion current and temperature for mass number, $m / e=2$.

types of poly-Si films, where the peak shift was defined as the difference between poly-Si and bulk Si $\left(521 \mathrm{~cm}^{-1}\right)$. FWHM and $\Delta \omega$ correspond to the defect density and internal stress of the poly-Si film, ${ }^{30)}$ respectively. The tensile stress that is observed at TO phonon wave numbers lower than $521 \mathrm{~cm}^{-1}$ is measured for the poly-Si film by the ELA method. The tensile stress increases in the order of $\mathrm{SiO}_{2}, \mathrm{SiN}$ and quartz substrates; defect density also shows nearly the same behavior. It was found that these phenomena are closely related with the appearance of the secondary grains. The tensile stress and the defect density decrease, as the growth of secondary grains becomes more frequent.

Next, the influence of hydrogen incorporation into the poly-Si film on the secondary grain growth is inferred. Figure 4 shows the relationship between the ion current and temperature for mass number, $m / e=2$. The ion current of the $\mathrm{SiO}_{2} / \mathrm{SiN} /$ glass substrate is larger than that of the glass substrate and almost equal to that of the $\mathrm{SiN} /$ glass substrate. The ion current corresponding to $m / e=1$, hydrogen atoms, is also detected during the increase of temperature. The hydrogen atoms or molecules contained in the $\mathrm{SiN}$ film are emitted to the vacuum above $300^{\circ} \mathrm{C}$. This result indicates that the hydrogen atoms or molecules can diffuse from the SiN film to melt-Si during ELA. The reason why the secondary grains are not frequently observed with the $\mathrm{SiO}_{2} / \mathrm{SiN} /$ glass substrate is the hydrogen incorporation into melt-Si during ELA from the SiN film. It is inferred that the hydrogen suppresses the secondary grain growth of poly-Si film. In the next sections, 3.2 and 3.3, the hydrogen behavior is examined quantitatively.

\subsection{Effect of hydrogen concentration on relation be- tween tensile stress and grain size of poly-Si film}

Figure 5 shows the relationship between the FWHM of the TO phonon peak and the energy density at 8 shots. The FWHM values are nearly constant for energy densities of 318 to $500 \mathrm{~mJ} / \mathrm{cm}^{2}$ and hydrogen concentrations of 2.3 and 4.2 at $\%$. The crystal defect density is nearly constant at 2.3 and 4.2 at\% of hydrogen, however, it increases steeply at $500 \mathrm{~mJ} / \mathrm{cm}^{2}$ and hydrogen concentration of $8.2 \mathrm{at} \%$. Figures 6(a) and (b) show the cross-sectional TEM micrographs for energy density of $500 \mathrm{~mJ} / \mathrm{cm}^{2}$ and hydrogen

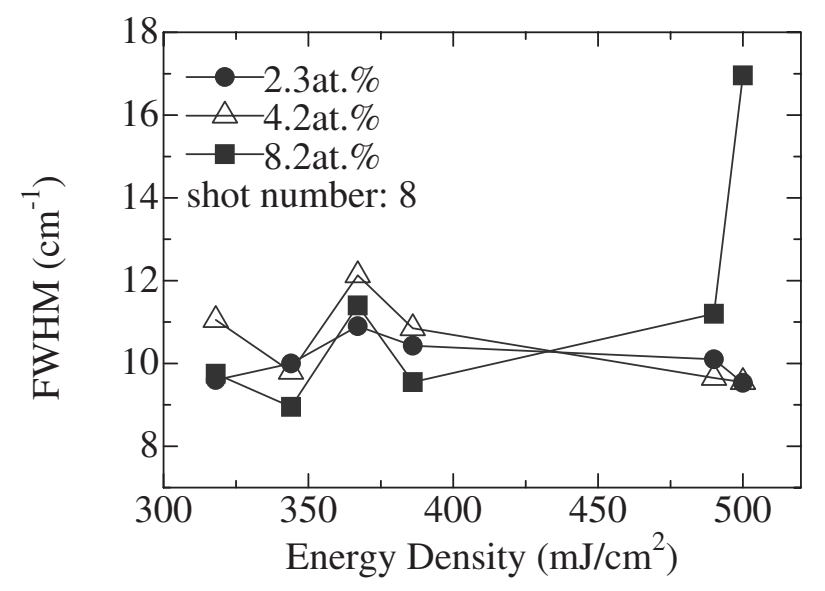

Fig. 5 Relationship between FWHM of TO phonon peak and energy density at 8 shots.

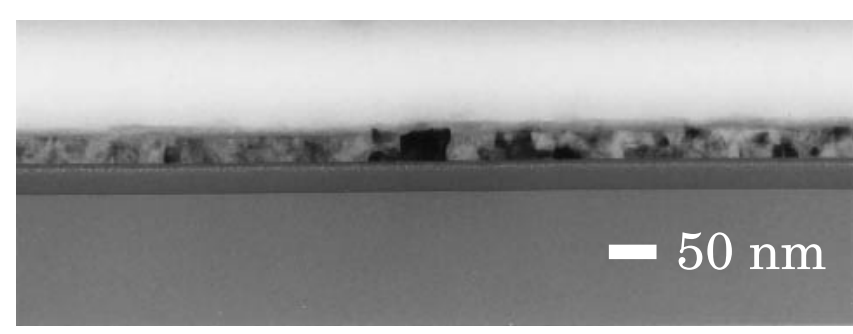

(a)

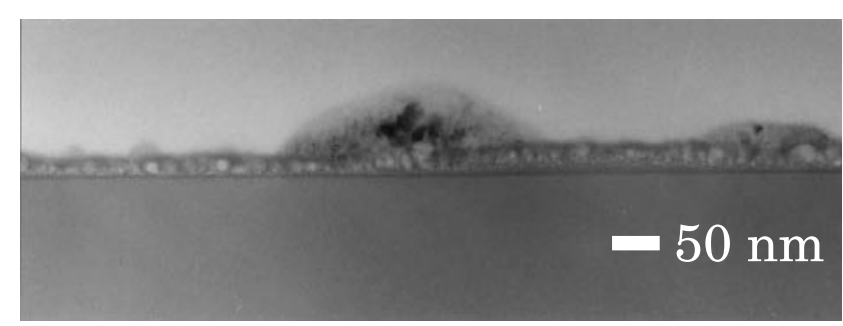

(b)

Fig. 6 Cross-sectional TEM micrographs for energy density of $500 \mathrm{~mJ} /$ $\mathrm{cm}^{2}$ and hydrogen concentrations of 2.3 at\% (a) and 8.2 at $\%$ (b).

concentrations of 2.3 and 8.2 at $\%$, respectively. For 8.2 at $\%$ hydrogen, $\mathrm{Si}$ atoms coalesce and form grains with sizes from $30 \mathrm{~nm}$ to approximately $600 \mathrm{~nm}$, although the poly-Si film is formed uniformly for 2.3 at $\%$ hydrogen. It is thought that some of the poly-Si films disappear by ablation which is caused by the hydrogen emission from $\mathrm{SiN}$ to vacuum via melt-Si during ELA for 8.2 at\% hydrogen. The reason why the FWHM of the TO phonon peak steeply increases at $500 \mathrm{~mJ} / \mathrm{cm}^{2}$ and hydrogen concentration of 8.2 at $\%$ is the hydrogen ablation. It is found that the effect of hydrogen introduced to melt-Si is very sensitive to the hydrogen concentration and the energy density. Figure 7 shows the relationship between $\Delta \omega$ and shot number. This experiment was conducted at $318 \mathrm{~mJ} / \mathrm{cm}^{2}$ to prevent hydrogen ablation during ELA. Although $\Delta \omega$ decreases rapidly with increasing 


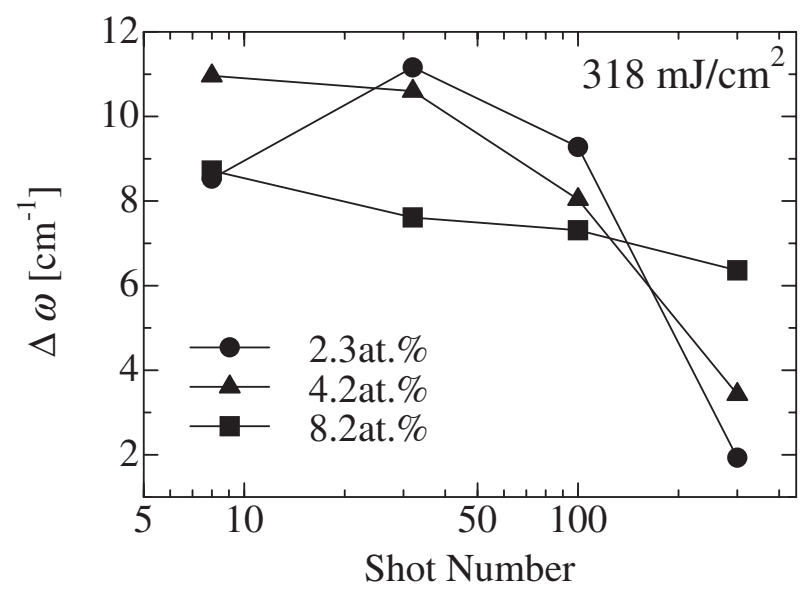

Fig. 7 Relationship between $\Delta \omega$ and shot number.

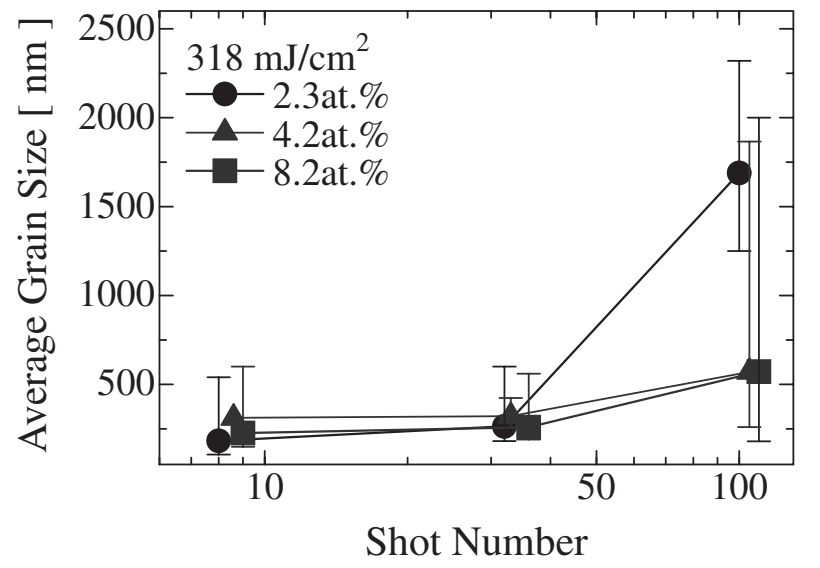

Fig. 8 Relationship between average grain size of poly-Si film and shot number.

shot number for 2.3 and 4.2 at $\%$, it decreases gradually with increasing shot number for 8.2 at $\%$ hydrogen. This result indicates that the stress relaxation of poly-Si with hydrogen concentration of 8.2 at\% in SiN film is smaller than that with 2.3 and 4.2 at $\%$ hydrogen. The phenomenon that the stress relaxation proceeds with increasing shot number for 2.3 and 4.2 at $\%$ hydrogen also indicates that the secondary grain growth proceeds with increasing shot number. ${ }^{25)}$ Figure 8 shows the relationship between the average grain size and shot number for $2.3,4.2$ and 8.2 at $\%$ hydrogen. The grain size becomes large with increasing shot number for each hydrogen concentration. For the condition of 2.3 at $\%$ hydrogen at 100 shots, the grain size rapidly increased and the maximum size exceeded $2 \mu \mathrm{m}$. Figure 9 shows the relationship between tensile stress and grain size for 4.2 and 8.2 at $\%$ hydrogen. The tensile stress decreases with increasing grain size from 200 to $600 \mathrm{~nm}$. It is found that, firstly, the tensile stress of the polySi film is released with a progress of secondary grain growth, and secondly, the stress relaxation becomes marked as the hydrogen concentration decreases.

Figures 10(a) and (b) show the SEM images for 2.3 and 4.2 at $\%$ hydrogen, respectively, with $318 \mathrm{~mJ} / \mathrm{cm}^{2}$ and 8 shots. The grain size of poly-Si with 2.3 and 4.2 at $\%$

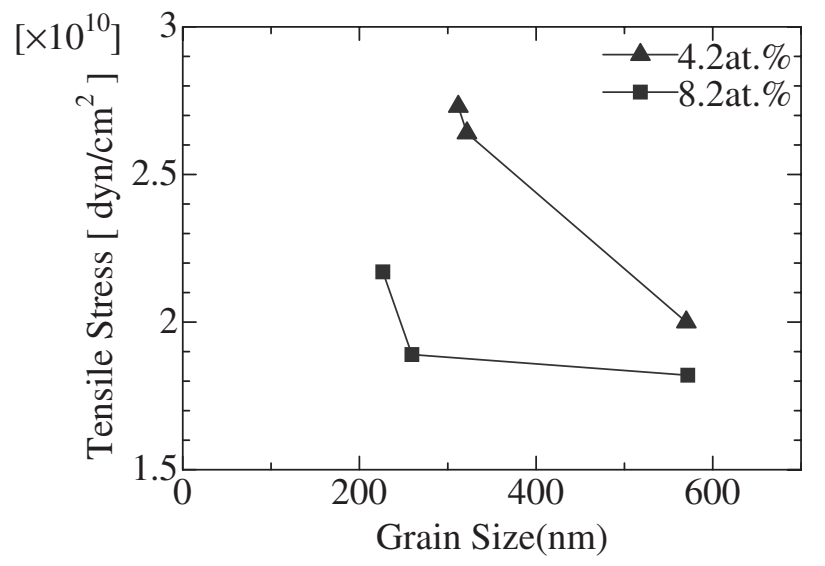

Fig. 9 Relationship between tensile stress and grain size.
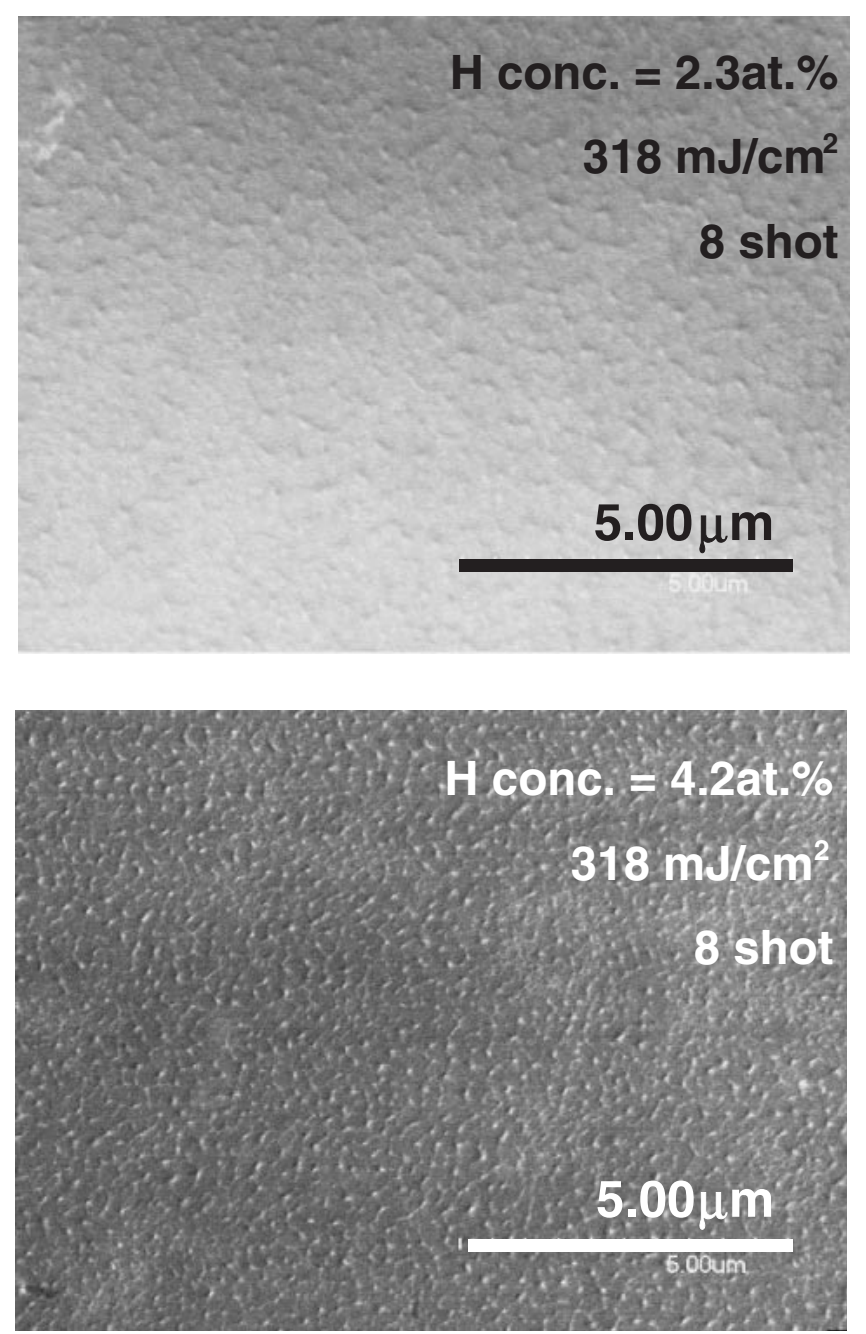

Fig. 10 SEM images of poly-Si films with hydrogen concentrations of 2.3 (a) and 4.2 at\% (b) for $318 \mathrm{~mJ} / \mathrm{cm}^{2}$ and 8 shots.

hydrogen are estimated to be 575 and $252 \mathrm{~nm}$, respectively. The grain size with 2.3 at $\%$ hydrogen is larger than that with 4.2 at\% hydrogen. From the results in Figs. 9 and 10, it is found that secondary grain growth is likely to occur, which causes stress relaxation at low hydrogen concentrations. This phenomenon fits the crystallization model based on the SPC, 


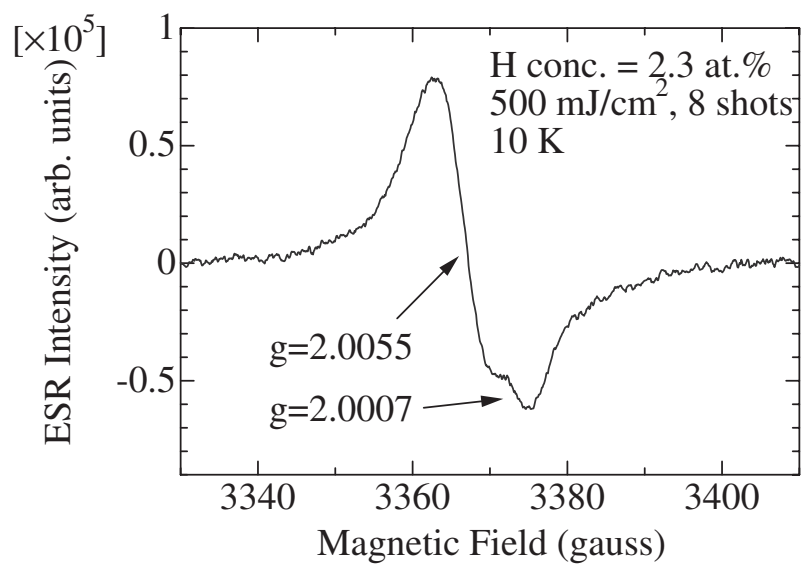

Fig. 11 ESR result for poly-Si film with $500 \mathrm{~mJ} / \mathrm{cm}^{2}$ and 2.3 at $\%$ hydrogen.

which we previously proposed, ${ }^{18)}$ in the point that secondary grain growth leads to tensile stress relaxation by the release of strain energy. The origin of the strain energy is thought to be the field of elastic stress around the dislocations at the grain boundary. The dangling bonds of the extra-half plane in the edge dislocation component at the grain boundary may be observed. In the next section, the spin density measured by ESR is discussed.

\subsection{Defect density in poly-Si film}

Figure 11 shows the ESR result at $500 \mathrm{~mJ} / \mathrm{cm}^{2}$ for 2.3 at $\%$ hydrogen. The signal of $g=2.0055$ and that of $g=2.0007$ correspond to the dangling bond in the poly-Si film and that in the quartz substrate, respectively. The spin density in the poly-Si film for a hydrogen concentration of 2.3 at $\%$ in $\mathrm{SiN}$ film is $2.1 \times 10^{19} \mathrm{~cm}^{-3}$. It is thought that the origin of the spin density is the crystal defects at the grain boundary, as we already pointed out. ${ }^{15)}$ Figures 12 (a) and (b) show the spin density in poly-Si films related to grain size with hydrogen concentrations of 2.3, 4.2 and 8.2 at $\%$ and that with hydrogen concentrations of 4.2 and 8.2 at $\%$, respectively. The solid line is the theoretically calculated result assuming that the defects are located at the grain boundary. ${ }^{15)}$ The spin density of polySi becomes low with increasing grain size for 4.2 and 8.2 at $\%$ hydrogen, which agrees with the theoretical values. This result indicates that the defects are located at the grain boundary, where the defect at the grain boundary is identified by the Si dangling bond. For 2.3 at\% hydrogen, the defect density exhibits a different behavior. We infer that a grain boundary without dangling bonds, such as the coincidence boundary, may be formed. These results indicate that the hydrogen concentration is closely related to defect formation. Figure 13 shows the schematic figure of the relationship between hydrogen and defect formation. Upper and lower figures are for high and low concentrations of hydrogen, and the left and right figures are before and during ELA, respectively. For high hydrogen concentration, transformation from $\mathrm{Si}-\mathrm{H}$ bonds to $\mathrm{Si}-\mathrm{Si}$ bonds via $\mathrm{Si}$ dangling bonds does not progress completely during ELA, and consequently, $\mathrm{Si}$ dangling bonds remain at the grain boundary. For low hydrogen concentration, $\mathrm{Si}-\mathrm{Si}$ bond formation progresses completely during ELA. These different mechanisms result

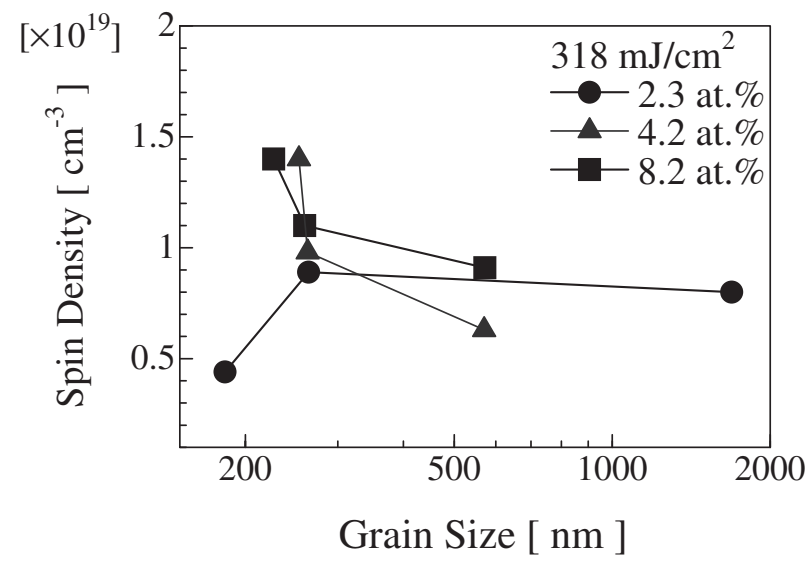

(a)

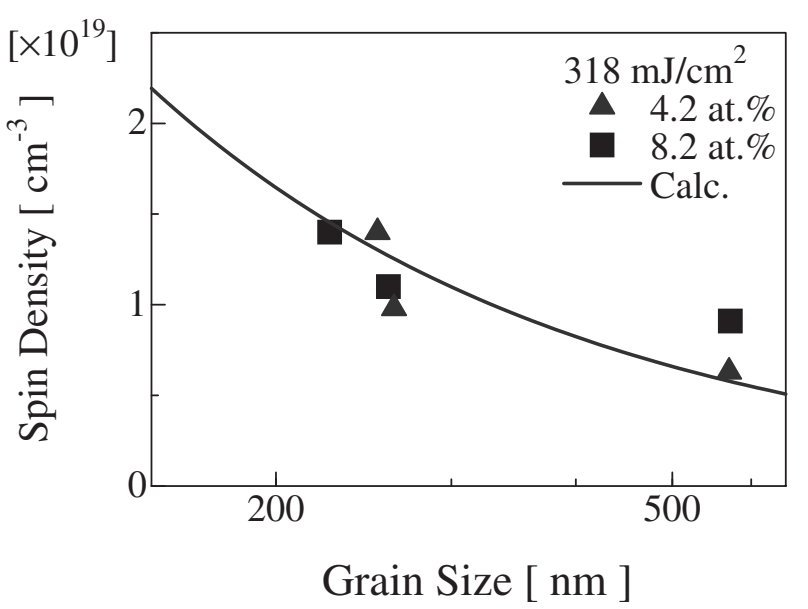

(b)

Fig. 12 Spin density in poly-Si films versus grain size with hydrogen concentrations of 2.3, 4.2 and 8.2 at\% (a) and 4.2 and 8.2 at\% (b). The solid line in (b) is the theoretical spin density.

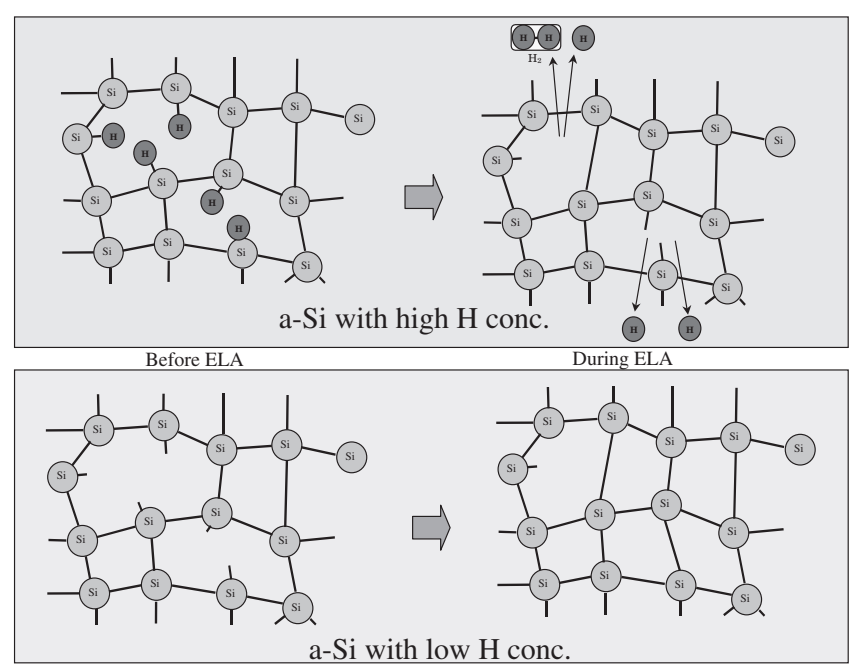

Fig. 13 Schematic figure of the relationship between hydrogen and defect formation.

in the different dependences of the spin density on the grain size. ${ }^{26)}$ This idea is in agreement with the present experimental data that spin density shows small values, but not with the calculated values for 2.3 at $\%$ hydrogen. 
Critical condition for secondary grain growth: Laser energy

+ Crystal defect energy at grain boundary (=strain energy) $=$ Critical value

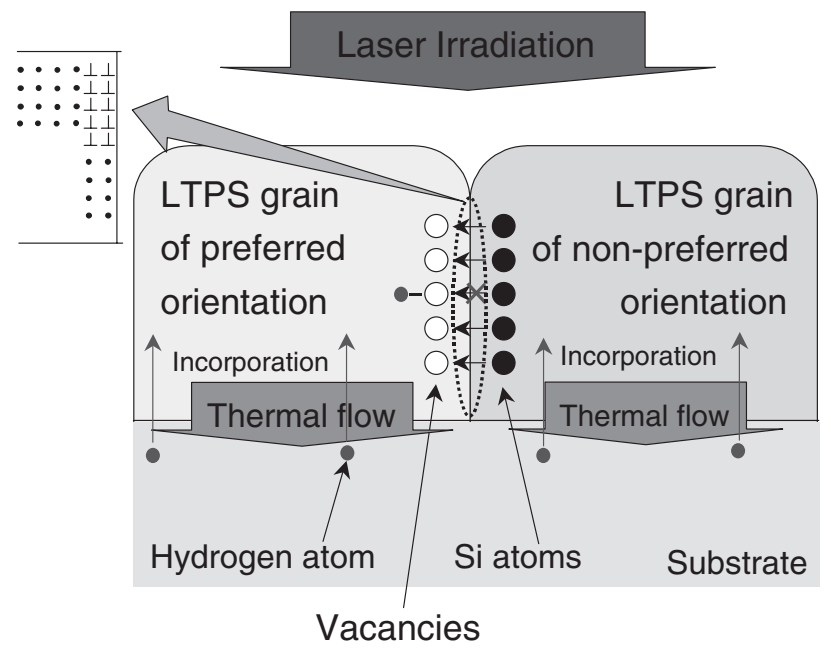

Fig. 14 Model of secondary grain growth by ELA for low energy density.

\subsection{Crystallization model}

The results described in the previous sections are clearly understood by using the following model. Figure 14 shows the model of secondary grain growth by the SPC. We already reported that grain enlargement of poly-Si under the solid phase occurs upon multishot laser irradiation at low energy density. The theory of secondary grain growth by the solid phase was studied by Thompson, ${ }^{31)}$ who assumed that the driving force of secondary grain growth is the energy of the grain boundary and film surface. Our proposed model also assumes the solid phase process. ${ }^{18)}$ The present model was constructed by taking into consideration the following experimental results. First, in-grain defects disappear upon laser irradiation because the defects move to the grain boundary. ${ }^{4,15)}$ It was also reported that the in-grain defect density is low for ELA poly-Si film compared with the SPC film after thermal annealing. ${ }^{32)}$ Second, rapid secondary grain growth occurs when the laser energy density and shot number reach critical values. ${ }^{16,17)}$ These phenomena indicate that secondary grain growth occurs when the sum of the laser energy and the crystal defect energy becomes a critical value. When a laser with the critical energy density is irradiated on a-Si at the critical shot number, the critical number of defects are integrated at the grain boundary. Simultaneously, the grain boundaries are melted, which lowers the activation energy of Si diffusion. Many $\mathrm{Si}$ atoms in a grain with nonpreferred orientation move to the vacancies in a grain with preferred orientation via the grain boundary. Then, grain boundary moves from the left to the right. Here, the vacancies are incorporated into Si due to the abrupt drop of temperature after laser irradiation is stopped.

According to the present model, the energy of strain generated by the dislocations at the grain boundary, is released at the start of secondary grain growth. The secondary grain growth and the tensile-stress relaxation, which proceeds through the secondary grain growth, occur simultaneously. It is assumed that the hydrogen atoms incorporated in the Si during the ELA form the hydrogenvacancy pair at the grain boundary and it prevents the secondary grain growth. Therefore, the grain size increases with decreasing hydrogen concentration. Our experimental results support the present growth model as follows. First, the tensile stress of the poly-Si film decreases with increasing grain size, as explained in Fig. 9. This phenomenon agrees with the model in the point that the secondary grain growth leads to the tensile-stress relaxation. Second, the diameter of poly-Si secondary grains with 2.3 at $\%$ hydrogen is larger than that with 4.2 at $\%$ hydrogen, as shown in Fig. 10. This phenomenon also agrees with the present model from the viewpoint of the effect of hydrogen on the grain growth. It has also been reported that the grain sizes of poly-Si with hydrogen concentrations of 1.3 and 2.8 at $\%$ are 500 and $150 \mathrm{~nm}$, respectively. ${ }^{33)}$ It seems to be reasonable that the hydrogen concentration in the Si film influences the grain size and the stress relaxation. The presented model does not apply to high concentrations of hydrogen, such as 8.2 at $\%$. Thus, for grain enlargement to occur upon ELA, it is important for the hydrogen concentration in the Si film to be decreased.

\section{Summary}

The poly-Si film formed by excimer laser irradiation of aSi film on glass or quartz substrates at low-energy density with multiple shots was characterized. The influence of secondary grain growth and the hydrogen supplied to melt-Si from the bottom SiN film on the tensile-stress relaxation of the poly-Si film was examined. The tensile stress in the polySi film relaxes with the appearance of the secondary grains. However, the hydrogen supply to melt-Si suppresses the secondary grain growth. It was also found that, firstly, the tensile stress of the poly-Si film decreases with increasing diameter of the secondary grains, and secondly, the stress relaxation becomes marked as the hydrogen concentration decreases. Furthermore, the relationship between the dangling bond density corresponding to the crystal defect density and grain size was investigated, and it was clarified that the crystal defects were located at the grain boundary for the hydrogen concentrations of 4.2 and 8.2 at $\%$, but the defect site was not identified for 2.3 at $\%$ hydrogen. Lastly, the recrystallization model by ELA at low-energy densities was discussed. The model was constructed based on the experimental data that secondary grain growth occurs at the critical energy density and the critical shot number, and it was shown that the present model was consistent with the results concerning the hydrogen concentration and secondary grain growth.

\section{Acknowledgements}

The author would like to thank Dr. Hiroki Hamada, SANYO Electric Co. Ltd., and Dr. Naoya Kawamoto, Yamaguchi University, for useful comments during the course of this study. The author also thanks Dr. M. 
Matsumura, Fellow of ALTEDEC Co. Ltd, for useful comments on the ELA method. Thanks are also due to Professor H. Matsumura and Dr. A. Masuda, JAIST, for preparing the Cat-CVD SiN film.

\section{REFERENCES}

1) R. S. Sposili and J. S. Im: Appl. Phys. Lett. 69 (1996) 2864-2866.

2) C. H. Oh, M. Ozawa and M. Matsumura: Jpn. J. Appl. Phys. 37 (1998) 492-495.

3) T. Sameshima, M. Hara and S. Usui: Jpn. J. Appl. Phys. 28 (1989) 1789-1793.

4) H. Hamada, H. Abe and Y. Miyai: Trans. IEICE J84 (2001) 65-75.

5) T. Noguchi, G. Kerrien, T. Sarnet, D. Debarre, J. Boulmer, D. Zahorski, M. Hernandez, C. Defranoux, C. Laviron and M. N. Semeria: Mat. Res. Soc. Symp. Proc., 717 (2002) pp. 33-38.

6) T. Ogawa, H. Tokioka, K. Furuta, Y. Satoh, M. Inoue, S. Yagi, M. Miyasaka, S. Inoue and T. Shimoda: Proc. 19th Int. Display Res. Conf., (1999) pp. 81-84.

7) A. Hara, F. Takeuchi, M. Takei, K. Suga, K. Yoshino, M. Chida, Y. Sano and N. Sasaki: Jpn. J. Appl. Phys. 41 (2002) 311-313.

8) M. Tai, M. Hatano, S. Yamaguchi, S. K. Park, T. Noda, M. Hongo, T. Shiba and M. Ohkura: Dig. Tech. Paper AM-LCD02, (2002) pp. 231234.

9) K. Tamagawa, H. Ikeda, T. Ohnishi, K. Kuwahara, M. Kikuchi, M. Hayama and K. Nakayama: Proc. IDW'03, (2003) pp. 585-588.

10) Y. Nakata, A. Shimoyanagi and S. Horita: Dig. Tech. Paper AMLCD2000, (2000) pp. 265-268.

11) V. Rana, R. Ishihara, Y. Hiroshima, D. Abe, S. Higashi, S. Inoue, T Shimoda, J. W. Metselaar and C. I. M Beenakker: Dig. Tech. Paper AM-LCD 03, (2003) pp. 17-20.

12) J. Jang, J. Y. Oh, S. K. Kim, Y. J. Choi, S. Y. Yoon and C. O. Kim: Nature 395 (1998) 481-483.

13) K. Makihara and T. Asano: Dig. Tech. Paper AM-LCD99, (1999) pp. 85-88.

14) T. Sadoh, I. Tsunoda, T. Nagata, A. Kenjo and M. Miyao: Dig. Tech. Paper AM-LCD’01, (2001) pp. 167-170.
15) N. Matsuo, A. Kinugasa, Y. Aya, T. Nouda, H. Hamada and T. Miyoshi: Trans IEICE J80 (1997) 245-248.

16) N. Matsuo, Y. Aya, T. Kanamori, T. Nouda, H. Hamada, A. Kinugasa and T. Miyoshi: J. Vac. Soc. Jpn. 41 (1998) 798-801.

17) N. Matsuo, H. Hamada, Y. Aya, T. Nouda and T. Miyoshi: J. Vac. Soc. Jpn. 42 (1999) 741-748.

18) N. Matsuo, Y. Aya, T. Kanamori, T. Nouda, H. Hamada and T. Miyoshi: Jpn. J. Appl. Phys. 39 (2000) 351-356.

19) N. Matsuo, H. Abe, N. Kawamoto, R. Taguchi, T. Nouda, H. Hamada and T. Miyoshi: J. Vac. Soc. Jpn. 43 (2000) 1120-1125.

20) N. Matsuo, H. Abe, N. Kawamoto, Y. Miyai and H. Hamada: Mater. Trans. 42 (2001) 2024-2025.

21) N. Matsuo, T. Nouda, N. Kawamoto, Y. Miyai and H. Hamada: Mater. Trans. 42 (2001) 2646-2648.

22) N. Kawamoto, H. Abe, N. Matsuo, Y. Miyai and H. Hamada: Trans IEICE J85 (2002) 659-665.

23) N. Matsuo, T. Nouda, N. Kawamoto, R. Taguchi, Y. Miyai and H. Hamada: Trans IEICE J85 (2002) 700-703.

24) N. Kawamoto, N. Matsuo, H. Abe, F. Anwar, I. Hasegawa, K. Yamano and H. Hamada: Jpn. J. Appl. Phys. 43 (2004) 293-298.

25) N. Kawamoto, N. Matsuo, A. Masuda, Y. Kitamon, H. Matsumura, Y. Harada, T. Miyoshi and H. Hamada: IEICE Trans. Electron. 88 (2005) 241-246.

26) N. Kawamoto, A. Masuda, N. Matsuo, Y. Seri, T. Nishimori, Y. Kitamon, H. Matsumura, Y. Harada, H. Hamada and T. Miyoshi: Proc. Intern. Display Workshop, (2004) pp. 495-496.

27) H. Matsumura: Thin Solid Films 395 (2001) 1-11.

28) A. Masuda, H. Umemoto and H. Matsumura: IEICE Trans 87 (2004) 203.

29) Y. Ikeda and T. Noguchi: Proc. 44th Symp. Semiconductor and Integrated Circuit Technology, (1993) p. 187.

30) K. Kitahara, A. Moritani, A. Hara and M. Okabe: Jpn. J. Appl. Phys. 38 (1999) 1312-1314.

31) C. V. Thompson: J. Appl. Phys. 58 (1985) 763-772.

32) H. Abe, Y. Aya, T. Nouda and H. Hamada: Dig. Tech. Paper AMLCD98, (1998) pp. 85-88.

33) Y. Yogoro, A. Masuda and H. Matsumura: Thin Solid Films 430 (2003) 296. 